\title{
After the epidemic: follow up study of HIV seroprevalence and changing patterns of drug use
}

\author{
C A Skidmore, J R Robertson, A A Robertson, R A Elton
}

\begin{abstract}
Objective-To follow up known intravenous drug users to determine current health state and drug use, compare characteristics with those of recent drug users, and examine HIV exposure and serostate.

Design-Subjects were identified from conventional general practice records and recruited from 1980 to the end of 1985; they were followed up during 1987 and 1988 and compared with drug users identified in the same way but recruited after 1985 .

Setting-General practice and community in north west Edinburgh. Follow up conducted throughout the United Kingdom.
\end{abstract}

Subjects-Subjects known to have injected illegal drugs before $1986(n=203)$ and since that time.

Main outcome measures-Mortality from and prevalence of HIV seropositivity and various parameters indicative of abstinence.

Results - Of the 203 subjects in the follow up group, $189(93 \%)$ were traced; $16(8 \%)$ had died and the remaining $173(85 \%)$ were interviewed. In all, 146 (72\% of the follow up cohort) had been tested for HIV antibodies, $94(64 \%)$ having positive and 52 $(36 \%)$ negative results; $57(28 \%)$ had not been tested. Of the 65 subjects in the recently recruited group, 51 (79\%) had been tested for HIV, $15(29 \%)$ having positive results. A further $21(43 \%)$ were currently negative for HIV antibody but still at risk. Thirty three $(19 \%)$ of those followed up were confirmed abstinent, although more (about half) showed evidence of diminished drug injecting. Age correlated strongly with abstinence $(p<0.001)$. One third of the group currently used cannabis, buprenorphine, dihydrocodeine, or diazepam. When the two groups were analysed together there was a strong association between the date of starting injecting and HIV seropositivity $\left(\chi^{2}=23.81, \mathbf{d f}=2, \mathbf{p}<0.001\right)$, with a peak around 1980-3.

Conclusions - Although only a fifth of the followed up group were convincingly abstinent, a much larger group showed evidence of prolonged periods of remission. Overall, much use of oral drugs was confirmed and worrying trends towards taking buprenorphine and benzodiazepines were evident. The peak incidence of starting drug use and the comparatively low seroprevalence of HIV in the newer drug users probably explain the anomalous high seroprevalence in Edinburgh drug users during 1980-5. The epidemic of HIV during the first half of the 1980s in the group suggests that the virus was probably being transmitted because of a pattern of behaviour. Changing patterns of HIV transmission suggest a need to concentrate on heterosexual transmission as the main problem in the future.

\section{Introduction}

Follow up studies of and observations on drug misuse have traditionally been conducted in centres of specialised care..$^{1-5}$ Many have used retrospective data or followed up people by using point incidence studies of drug taking. Interest naturally centres on mortality and numbers still using various drugs after the elapse of several years or decades, ${ }^{67}$ but most studies recognise the difficulties in trying to evaluate changes in lifestyle that may have led to abstinence other than the vague notion of "maturing out" of drug use. ${ }^{8}$

The enormous difficulties in drawing conclusions from small amounts of information have to a large extent allowed confusion to prevail in establishing effective and lasting policies for managing drug users. The need for a more profound understanding of drug use has become more evident in the late 1980 s, perhaps because of HIV infection and the extension of drug injecting to the provinces and into a wider social range of people. The importance of relapse rather than remission, ${ }^{410}$ the need for recurrent detoxification rather than single treatments, and the establishment of damage limitation or risk reduction as a policy worth pursuing when abstinence is not likely make the need for information about lifestyles and changes in drug taking more necessary.

Critically, the realisation that drug users attending specialist clinics represent only the tip of the iceberg has highlighted throughout the 1980s the need for more information about those not attending for advice or help. In the United States particularly and to a lesser extent in Europe the decreasing distinction between hard and soft drugs and the importance of extending education to all those who might ever inject have been brought about by AIDS. In the United Kingdom recent recommendations include accepting the reality of drug use rather than forever hoping that it will go away with legislation and control. 112

The present data relate to a group of people attending a non-specialist centre (a general practice in Edinburgh) for help for a drug related problem. All were intravenous drug users and most were identified within a comparatively short time after starting to inject. Though half of them continued to attend the same general practice, the follow up study includes those currently registered elsewhere; many were contacted throughout the United Kingdom, often afte many years had elapsed.

This study was therefore established not just to measure mortality as an outcome or to identify the point at which cure is present but to try to judge prognostic factors that might indicate favourable outcome and patterns of drug use. The recognition that return to drug use often happens after years of abstinence made it more interesting to concentrate on patterns of drug use and changing behaviour rather than simply on whether the subject was taking drugs.

Several things make it a unique study. It is an observation of a group in the community with no specialist care necessarily being present. ${ }^{13}$ The subjects were largely recruited early in their drug taking rather than after years of drug use ${ }^{10}$ and consequently are

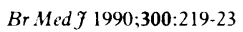

Edinburgh Drug Addiction
Study, Muirhouse Medical
Group, Edinburgh EH4
4PL
C A Skidmore, MA, research
associate
J R Robertson, FRCGP,
general practitioner
A A Robertson, MRCGP,
general practitioner
Medical Statistics Unit,
University of Edinburgh,
Edinburgh EH8 9AG
R A Elton, PHD, senior
lecturer
Correspondence to: Dr J R
Robertson. 
young compared with those in many comparable studies. Finally, they are perhaps the first cohort which has been devastated by HIV infection. ${ }^{1+}$ The epidemiological importance of HIV in this group was examined by studying the spread in a cohort and comparing it with that in a second group who started injecting some years later.

\section{Methods}

Over six years 203 subjects known to have injected illegal drugs were recruited into a prospective study of patterns of drug taking. This cohort was closed in December 1985, and subsequent people found to be injecting drugs were recruited in the same way to another group for comparison. There were 65 subjects in this later group. Basic data were recorded during the course of consultations in general practice, and all patients were flagged at the office of the registrar general for Scotland to enable follow up and subsequent notification of deaths.

Follow up was started in January 1987 and finished in December 1988; whenever possible interviews were carried out in face to face consultations. When patients were unable to attend the surgery they were contacted in their homes, many interviews being conducted outside Edinburgh. In all cases in which patients were still registered with the same practice consent was obtained from the general practitioner before an interview was carried out. For those no longer registered with this practice we established contact by determining the current general practitioner through the central register and the appropriate health board (Scotland) or family practitioner committee. His or her advice and consent were taken in approaching the patient. Interviews were conducted by two of us, one in the practice (CAS) and the other in the homes of subjects no longer attending the practice (JRR). When possible a sample of blood was taken by venesection and analysed for HIV antibody and antigen, with CD4 counts and full blood counts being performed. Interviews consisted of a structured questionnaire covering five main topics and 217 variables. The five topics were background demographic details, previous drug use, present pattern of drug use, knowledge of AIDS and risk behaviour, and general health.

Those not available for interview were included as respondents if their current general practitioner was prepared to complete a modified questionnaire briefly

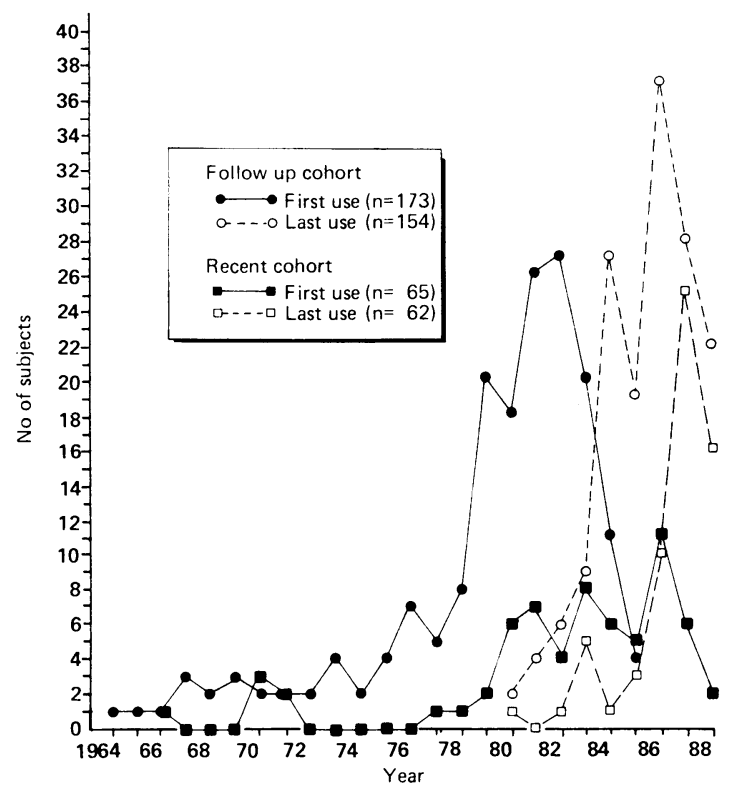

Year of first and last use of drugs in follow up cohort and more recently recruited cohort. Data are missing for year of last use in both groups covering the same topics of interest. In all cases an additional form was completed by the researcher to include data gathered subsequent to interview, validation of self reported information by discussion with the current general practitioner, and laboratory results when available. This was completed four months after the interviews had been completed to cover subsequent episodes of drug injecting.

The whole cohort (268) was analysed by date of first starting drug use to explore the relation between this variable and the presence of HIV antibodies.

All these data were coded and entered on the Edinburgh University mainframe computer and analysed using software of the statistical package for the social sciences (SPSS). Two way frequency tables were analysed by $\chi^{2}$ tests for two by two tables. Groups were compared for quantitative variables by Wilcoxon rank sum tests, and Kendall rank correlation was used for testing association between quantitative variables. The relation between HIV seropositivity and year of first drug use was analysed by logistic regression, with a quadratic term being entered to fit the pattern of a peak in prevalence.

\section{Results}

\section{FOLLOW UP COHORT}

The 203 subjects recruited to the study over the six years comprised 140 men and 63 women. All had injected drugs intravenously, although two denied any such behaviour when interviewed at follow up. A total of 189 were traced by various means, 173 being interviewed and 16 having died. The remaining 14 were untraced for various reasons: four had been seen recently by their general practitioner, one was thought to be using an alias, and the remaining nine were lost because of frequent changes of address or failing to register with any services. Those lost to follow up had a mean age of 31 and all but one were men. Most had started using drugs before the peak years of HIV transmission.

The mean age of those interviewed was 29.6 years (mode 25 years); $113(65 \%)$ were men and $60(35 \%)$ women. In all, $151(87 \%)$ had been born in Edinburgh and $137(79 \%)$ were unemployed at follow up. Thirty three (19\%) were married and a further $50(29 \%)$ cohabiting; $102(59 \%)$ had one or more children (total number of children 201$)$. Thirty five $(20 \%)$ were living with their parents. Over half $(94,54 \%)$ were still registered with this practice and were seen on the premises or at home.

The remaining $79(46 \%)$ were traced through health board records to Scottish prisons or other general practices throughout Scotland. Six subjects were interviewed in England. Two had subsequently emigrated to North America. A total of 112 traced subjects $(59 \%)$ were found to have been in prison since 1980 , most between 1985 and 1986. Men were more likely to have been in prison than women.

The length of drug use was on average 7.5 years (range 1-23, mode 7 years), although the peak years for starting to use drugs were 1981 and 1982 (see figure). The most common drugs of current use were oral dihydrocodeine and diazepam (59 and 58 of the 173 subjects respectively). Ninety three subjects (54\%) took the drugs by injection at the first use. In all, 146 subjects (84\%) had been tested for HIV antibodies, 94 having positive and 52 negative results; 57 of the 203 subjects had not been tested.

Abstinence (no injection of drugs for more than 12 months) was recorded in self reports from 82 subjects $(47 \%)$ but was confirmed for only $33(19 \%)$ when additional data were considered (table I). These additional data included interviews with the subjects' general practitioners, results of urine tests, and sub- 
TABLE I-Abstinence (no injection of drugs in previous 12 months or more) in follow up cohort

\begin{tabular}{lc}
\hline & No of subjects \\
\hline Abstinent on main questionnaire & 82 \\
And by additional assessment $\dagger$ & 33 \\
But not on additional assessment $\dagger$ & 49 \\
Abstinent on additional assessment but not on & 8 \\
main questionnaire & 83 \\
Not abstinent on both criteria & 173 \\
\hline Total &
\end{tabular}

*Self report.

tUrine test results, interview with subject's general practitioner, subsequent data, hospital letters, etc.

sequent performance over a period of several months.

Although most subjects $140(81 \%)$ were still injecting drugs, only 25 of them (18\%) said that they were currently using heroin. Many continued to inject other drugs, principally dipipanone hydrochloride, buprenorphine, and amphetamine, and to use oral drugs frequently (table II). Details about drug use in the previous six months showed that 110 of the cohort (64\%) were abstinent and only $35(21 \%)$ used drugs weekly or more regularly.

To explore any relation between age and patterns of drug use the 173 subjects were divided into arbitrary categories by year of birth: before and including 1955 , 1956-60, 1961-5, and 1966 and later. Those born in 1961-5 were more likely to be using heroin $(p<0.01)$, cocaine $(\mathrm{p}<0.05)$, amphetamine $(\mathrm{p}<0.05)$, barbiturates $(\mathrm{p}<0.05)$, buprenorphine $(\mathrm{p}<0.01)$, and dihydrocodeine $(p<0.05)$. They were significantly more likely to be positive for HIV ( $p<0 \cdot 01$; table III), although the prevalences of illnesses such as hepatitis, septicaemia, and thrombosis were not different between groups. They were also more likely to have shared needles and syringes $(93 \%$ (45/48) compared with $84 \%$ $(31 / 37) ; \mathrm{p}<0.05)$.

Age was found to correlate with abstinence $(\mathrm{p}<0.001)$, the older users being more likely to be abstinent. Age was not found to be associated with sex in the different groups.

The use of agencies, specialist or general, was found to be similar in all groups with the exception of drug dependency units, which were used much more often by older subjects $(\mathrm{p}<0.001)$.

TABLE III - Numbers of subjects positive for $H I V$ by year of birth

\begin{tabular}{lc}
\hline Year of birth & $\begin{array}{c}\text { No of subjects } \\
\text { positive for HIV }\end{array}$ \\
\hline-1955 & 12 \\
$1956-60$ & 25 \\
$1961-5$ & 47 \\
$1966-$ & 10 \\
\hline Total & 94 \\
\hline
\end{tabular}

\section{RECENTLY RECRUITED (CONTROL) COHORT}

Sixty five subjects were identified as being intravenous drug users, but they were not part of the cohort recruited from 1980 to 1986 . They were established as a control group, comprising 47 men $(72 \%)$ and 18 women $(28 \%)$. The average age of the group was $25 \cdot 3$ years (range $18-38$, mode 22 ). Sixty $(92 \%)$ had been born in Edinburgh and $62(95 \%)$ in Scotland as a whole. Thirty one $(48 \%)$ were married or cohabiting

TABLE IV - HIV state and year of first use of drugs in whole cohort $(n=268)$. V'alues are numbers of subjects

\begin{tabular}{|c|c|c|c|c|c|}
\hline \multirow[b]{3}{*}{ Year of first use } & \multirow[b]{3}{*}{ Positive } & \multicolumn{2}{|c|}{ HIV state } & \multirow[b]{3}{*}{ Not tested } & \multirow[b]{3}{*}{ Total } \\
\hline & & \multicolumn{2}{|c|}{ Negative } & & \\
\hline & & Still at risk & No longer at risk & & \\
\hline-1970 & 4 & & 6 & 4 & 14 \\
\hline $1971-5$ & 4 & 2 & 7 & 1 & 14 \\
\hline $1976-8$ & 9 & 3 & 3 & $i$ & 16 \\
\hline 1979 & 13 & 2 & 5 & 2 & 22 \\
\hline 1980 & 12 & 4 & 5 & 2 & 23 \\
\hline 1981 & 20 & 3 & 2 & 3 & 28 \\
\hline 1982 & 20 & 6 & 2 & 2 & 30 \\
\hline 1983 & 16 & 3 & 5 & 2 & 26 \\
\hline 1984 & 4 & 7 & 2 & 1 & 14 \\
\hline 1985 & 7 & 11 & 8 & 2 & 28 \\
\hline 1986 & & 2 & & & 2 \\
\hline Total & 109 & 43 & 45 & 20 & $217^{\star}$ \\
\hline
\end{tabular}

*HIV state of remaining 51 subjects was unknown. and had children. Fifty six $(86 \%)$ were unemployed at the time of interview, and $37(57 \%)$ had been in prison at some time. The average number of siblings was 3.6 , $22(34 \%)$ subjects having a brother or sister who also used drugs.

The average length of drug use for the group was 5.5 years (range 1-21, mode 1 year). The most common drug currently taken was dihydrocodeine ( 37 subjects, $37 \%)$ followed by buprenorphine $(29,45 \%)$ and diazepam $(24,37 \%)$. Twenty nine $(44 \%)$ subjects said that their first use of drugs had been injecting heroin and that they had injected drugs outside Edinburgh, while nearly half the subjects $(31,48 \%)$ said that most of their friends also used heroin and most $(52,80 \%)$ knew someone who had died of drug use.

Fifty one (79\%) subjects had been tested for HIV, of whom 15 were positive for HIV antibody; a further 23 subjects were currently negative for the antibody but were reported to be still indulging in risk taking behaviour.

WHOLE COHORT $(\mathrm{N}=268)$

The data in table IV show the relation between HIV seropositivity and the year when drugs were first used. Quadratic logistic regression showed that timing was highly significant $\left(\chi^{2}=23.81, \mathrm{df}=2, \mathrm{p}<0.001\right)$, the peak of HIV seropositivity occurring around 1980-1.

\section{COMPARISON OF TWO GROUPS}

The data were analysed to highlight any differences or similarities between the two groups in the study the follow up group $(n=173)$ and the control group of recent users $(n=65)$.

The two groups were found to be different in terms of age, with the follow up group on average $3 \cdot 2$ years older $(26.7 v 24.4$ years; $\mathrm{p}<0.001)$. This age difference was reflected in the average length of drug use for both groups, the follow up group having an average use of 7.5 years (range $1-23$ ) compared with 5.5 years (range $1-21)$ in the more recent users $(p<0.01)$. The figure shows the distribution of year of first and last use for both groups. No differences were found in the sex distribution between the two groups, women accounting for $35 \%(60)$ of the follow up group and $28 \%$ (18) of the recent users.

The choice of drugs used differed between the two groups. Recent users were significantly more likely to use buprenorphine $(\mathrm{p}<0.05)$, dihydrocodeine $(\mathrm{p}<0 \cdot 01)$, and temazepam $(\mathrm{p}<0 \cdot 01)$. All subjects were questioned about which drug or drugs they had begun to use most recently. Two fairly distinct patterns emerged with a Wilcoxon rank sum test. The follow up cohort was significantly less likely to have started using any drugs recently $(\mathrm{p}<0.001)$ and the younger more recent users were more likely to have begun using buprenorphine and dihydrocodeine. No significant differences emerged between the groups in the use of heroin, methadone, cocaine, solvents, amphetamines, barbiturates, cannabis, or lysergide.

The two groups showed different patterns of drug misuse, particularly during the previous six months. In all, $41 \%$ (26) of the recent users said that they were currently using drugs regularly (defined as more than three times a week) compared with only $12 \%(20)$ of the follow up group. At the other end of the scale, $65 \%$ (112) of the follow up cohort $v 41 \%$ (26) of the recent group were likely to have been abstinent for three months or longer, both being significant at $\mathrm{p}<0.001$ with a Wilcoxon rank sum test.

Abstinence was examined between the two groups by using several variables. Subjects were asked early in the questionnaire when they had last injected heroin. Heroin later became synonymous for any drug as heroin itself became scarce. With a Wilcoxon rank sum 
test recent users were more likely than the followed up users to have last injected heroin in 1987 and 1988 Later subjects were asked how long they had been abstinent and the date of their last intravenous injection. Recent users were significantly more likely to have a short length of abstinence (less than four weeks or one to six months) than the followed up users (two to five years, $\mathrm{p}<0.001$ ). In addition, recent users were more likely than the followed up group to mention 1987 and 1988 as the year of last injection $(p<0 \cdot 01)$.

A similar proportion of subjects in both groups had been tested for HIV ( $84 \%$ (146) of those interviewed in the follow up cohort and 79\% (51) of the recent users), but $64 \%$ (94) of the follow up cohort compared with only $29 \%(15)$ of the recent users were infected with HIV. When questioned closely about how much they had previously shared needles $89 \%$ (155) of the follow up group said that they had shared needles compared with $62 \%(40)$ of the recent users $(p<0 \cdot 001)$.

The two groups were compared to see whether any differences emerged in the type or amount of help sought for problems related to drug misuse. The results of a series of $\chi^{2}$ tests showed that the followed up cohort were more likely to have sought help from their general practitioner $(\mathrm{p}<0 \cdot 10)$, a drug dependency unit $(\mathrm{p}<0.01)$, or a voluntary agency $(\mathrm{p}<0.001)$ and were more likely to have been in a residential establishment $(\mathrm{p}<0 \cdot 05)$. They were also significantly more likely to have been in prison than the more recent group $(p<0 \cdot 05)$. Both groups cited their general practitioner as the most useful source of help, in fact more often than any other source $(\mathrm{p}<0 \cdot 10)$.

\section{Discussion}

The timing of the wave of new drug users in the Edinburgh locality probably coincides with the introduction of HIV into this group quite closely and therefore suggests that the high seroprevalence may result from this coincidence. Those starting drug use during this time were additionally more likely to have shared needles, for whatever reason, further increasing the chances of infection. The previously noted high seroprevalence ${ }^{14}$ has now been shown to be higher and may be over $80 \%$, assuming a similar high rate in those still untested and removing the few never apparently at risk.

The comparative sparing from HIV infection of both older and younger people is interesting. Presumably needle sharing was and is less common outside these two age groups, availability partly accounting for this difference in behaviour. The older drug users had the advantage of a drug dependency unit, and the younger ones may have had the advantage of knowledge and information about AIDS. The many who are infected, as well as their cohabitants and children, are the immediate problem for all medical and social services in Edinburgh and evidently elsewhere in the United Kingdom. Although the seroprevalence may increase with time in the younger drug users, most of them have now had several tests over a prolonged period and remain negative for HIV antibody. In fact the small proportion found to be seropositive seem to be part of the larger cohort when their time of first use of drugs is considered.

Despite this the combined cohort seems otherwise to be similar to other groups studied in that older subjects are more likely to be abstinent and many subjects have episodes of abstinence and relapse. The trend towards reduced drug injecting over time may be important for planning treatment and interpreting the results of treatment. The similarities continue in the experience of drug related diseases, with the exception of HIV infection. Demographic characteristics such as employment (or lack of), socioeconomic state, and educational background are similar to those in other study groups but some findings distinguish our cohort. The subjects were generally younger than those in most studies in the United States and were mainly born and living locally. The finding that many lived with parents is of importance and again is different from many national and international studies. ${ }^{15}$ It may reflect the shift of drug use towards areas of social deprivation. Those lost to follow up were largely older, which may partly explain their comparative inaccessibility. Only two were known to be positive for HIV, although the remainder had not been recently tested.

The relation between HIV testing and knowledge of HIV serostate and continued drug use was unclear. No convincing evidence was available to suggest that those who had been tested, with positive or negative results, were more or less likely to be abstinent. Many examples were found of subjects who injected more because of having a positive test result as well as those who abstained in these circumstances. Unfortunately, many examples of those who were repeatedly seronegative and continued to inject (and share equipment) are evident.

The local and national campaigns have increased since 1985, when, largely as a result of the findings in Edinburgh, drug users and by implication heterosexuals were recognised as a major risk group. In 1985 the test (HIV antibody) was made available and applied to blood transfusion donations and clinically to drug users. In 1986 a national campaign in the press, on posters, and in leaflets sent to every household was targeted at the whole population. In 1988 a national campaign targeted specifically at drug users employed similar tactics. The local network and health education activities have been intense during this time, perhaps providing more appropriate information for local consumption. It is of some interest that local workers recognise the need to constantly update and even change the message as new concerns become obvious. This particularly relates to changing drug taking patterns and the awareness of many sexual partners being at risk of HIV infection.

In all respects, therefore, this group seems to conform to expectations about mortality, morbidity (with the exception of HIV), and abstinence. HIV and AIDS distinguish the cohort, and the trend towards new drugs of abuse raises new concerns. The strong association of a wave of drug use and clinical problems, in this case HIV, is similar to waves of drug misuse in recent years that resulted in epidemics of hepatitis $\mathrm{B}$, endocarditis, candidal endophthalmitis ${ }^{16}$ and other infections. Behavioural characteristics, availability of drugs, and the presence of a source of infection are clearly the driving forces in the HIV epidemic. The strong relation between seropositivity and year of first drug injection suggests that needle sharing and perhaps sexual patterns were distinctly different in those years. This is supported by clinical observations and self reported information. Resources and further research should be directed to identify and divert similar emerging problems elsewhere.

Despite our suggestions that the epidemic phase of HIV transmission was over by 1984 , at least in this part of the Edinburgh drug using community, cases of AIDS are still few. Only three of the deaths have been directly attributed to AIDS. In the immediate future, however, mortality is likely to escalate rapidly despite the comparatively controlled drug use. The wave of cases of AIDS must not be allowed to obscure these changes in drug misuse or to confuse the need for services directed at drug problems as well as AIDS. ${ }^{17}$ Drug misusers and maybe unprotected heterosexual intercourse must command most urgent attention.

We thank the partners and staff of the Muirhouse Medical 
Group for their support and dedication in providing data and advice. The many general practitioners and family practitioner committees who now have responsibility for some of the patients were a resource without which the follow up would not have been possible. The chief scientist office of the Scottish Home and Health Department has supported the project enthusiastically for several years.

1 Blumberg HH. British users of opiate-twpe drugs: a follow up study, $B r f$ Addict 1976:71:65-77.

2 Stimson GV, Oppenheimer E, Thorley A. Seven-year follow-up of heroin addicts: drug use and current outcome. Br Med f 1978:i:1190-2

Vaillant G. A 20-year follow-up of New York narcotic addicts, Arch Gen Psychiatry 1973:29:237-41.

4 Chapple PAL, Somekh DE, Taylor ME. Follow-up of cases of opiate addiction from the time of notification to the Home Office. Br Med f 1972;ii:680-3.

5 Wille R. Ten year follow-up of a representative sample of London heroin addicts, clinic attendance, abstinence and mortality. Br $\mathcal{F}$ Addict 1981; 76:259-66.

6 Bewley TH, Ben-Arie $\mathrm{O}$. Morbidity and mortality from heroin dependence: study of 100 consecutive in-patients Br Med J 1968;i:727-30.

Ghodse AH, Sheenan M, Taylor C, Edwards G. Deaths of drug addicts in the United Kingdom 1967-1981. Br Med f 1985;290:425-8.
8 Winick C. Maturing out of narcotic addiction. Bulletin of Nurcotics 1962:14

9 Marlat GA, George WH. Relapes prevention and overview of the model. $\mathrm{Br} y$ ddict 1984;79:261-73.

10 Robertson JR, Bucknall ABV, Skidmore CA, et al. Remission and relapse in heroin users and implications for management: treatment contol or risk reduction. Int $\mathcal{F}$ Addict 1989;24:229-46.

11 Department of Health and Social Security. AIDS and drug misuse part l: report of the Advisory Council on the Misuse of Drugs. London: HMSO, 1988.

12 Department of Health. AIDS and drug misuse part 2: report of the Adrison Council on the Misuse of Drugs. London: HMSO, 1989.

13 Roberts JJK, Skidmore CA, Robertson JR. Human immunodeficiency virus in drug users and increased consultation in General Practice. I R Coll Gen Pract 1989:39:373-4.

14 Robertson JR, Bucknall ABV, Welsby PD, et al. Epidemic of AIDS related virus ( $\mathrm{H} T \mathrm{LV}$-III/LAV) infection among intravenous drug abusers. Br.Med 1986:292:527-9.

15 Stimson GV, Oppenheimer E. Heroin addiction: treatment and control in Britain. London: Tavistock, 1982

16 Robertson JR. Heroin, AIDS and society. London: Hodder and Stoughton, 1987.

17 Robertson JR, Skidmore CA. AIDS in the family: the second report of th Edinburgh drug addiction study. Edinburgh: Scottish Home and Health Department, 1989.

(Accepted l December 1989)

\title{
Making better decisions: construction of clinical scoring systems by the Spiegelhalter-Knill-Jones approach
}

\author{
D G Seymour, M Green, F G Vaz
}

\begin{abstract}
A study was carried out assessing the practical use of a simple system of scoring information which can help in making a diagnosis or establishing a prognosis in an individual patient. The system was introduced in 1984 for use in gastroenterology, but it can be employed in a wide range of medical and surgical settings. This series was concerned with predicting postoperative respiratory complications in a group of elderly surgical patients.
\end{abstract}

The system combines elements from Bayes's theorem and logistic regression, though no mathematical knowledge is required to apply it in clinical practice. The method by which results are presented is easy to understand, yet at the same time more complex ideas such as conflict of evidence and doubt may be embraced if the clinician so desires.

\section{Introduction}

Weighing up signs, symptoms, and test results in an individual patient, comparing them with past experience, and arriving at a reasoned decision lie at the heart of clinical medicine. Recently Spiegelhalter and Knill-Jones have proposed a simple scoring system which can add precision to risk assessment in individual patients. ${ }^{1-4}$ To the non-mathematical user the great attraction of the system is that it demands only the ability to add, subtract, and look up a reference table or graph. Despite its simplicity, however, the system has a firm statistical basis and can handle complex diagnostic concepts such as conflict of evidence and doubt. ${ }^{1}$ The system can be used equally well to establish prognosis or to estimate the likelihood of a particular diagnosis (as in the original application). ${ }^{\prime}$ This paper examines the system in relation to establishing prognosis.

The Spiegelhalter-Knill-Jones approach has been welcomed by many statisticians (see discussion after Spiegelhalter and Knill-Jones') because of the elegance with which it combines elements of Bayes's theorem with those of logistic regression. The result is a system which neatly sidesteps some of the main disadvantages of the two original techniques. For example, it does not assume that all risk factors are acting independently within each outcome class (the "independence Bayes" assumption, which is central to many bayesian analyses), while at the same time predictions are presented in a form which is less mathematical and much more clinically relevant than the output of conventional logistic regression analysis.

We believe that the Spiegelhalter-Knill-Jones system deserves to be better known in clinical medicine, and this paper therefore focuses on the practicalities of using the technique rather than on its statistical origins. We refer to these, however, in the appendix and discussion.

\section{Present study and results}

SOURCE OF ILLUSTRATIVE DATA

All patients who served in the assessment were aged 65 or over and received a general surgical operation. They comprised 258 patients from Dundee (age range 65-93) and 299 patients from Cardiff (age range 65-97). Two thirds of the patients in both data sets were aged between 65 and 74 . Full details of the patients are given elsewhere.

Many variables relating to preoperative state and postoperative outcome were collected prospectively in both data sets by using criteria established in advance and which are described elsewhere. ${ }^{67}$ As our purpose is to illustrate the Spiegelhalter-Knill-Jones approach with a simple clinical example, this paper looks simply at the ability of four preoperative risk factors (preoperative chest disease, smoking in the six weeks before admission, site of the surgical incision, and presence of volume depletion) to predict postoperative respiratory complications. Full details of how variables were defined in the larger study and of how these four respiratory variables were selected from an initial list of 19 have been reported. ${ }^{5-7}$ Brief definitions are given in table I.

The collection of two data sets, the first from Dundee and the second from Cardiff, was deliberate. Data collection from both data sets was completed before any calculations were performed, but the weightings on which the Spiegelhalter-Knill-Jones system is based were calculated solely from the patients from Dundee, who thus made up the "training" data set. The weightings obtained from the training data set were then applied to the Cardiff group - that is, the
Correspondence to: Dr Seymour. 